\title{
Skeletal Muscle Mass Influences Tolerability and Prognosis in Hepatocellular Carcinoma Patients Treated with Lenvatinib
}

\author{
Haruki Uojima ${ }^{a, b}$ Makoto Chuma ${ }^{c}$ Yoshiaki Tanaka ${ }^{a}$ Hisashi Hidaka ${ }^{a}$ \\ Takahide Nakazawaa, d Shogo Iwabuchie Satoshi Kobayashif \\ Nobuhiro Hattorig Katsuaki Ogushic Manabu Morimoto ${ }^{f}$ \\ Tatehiro Kagawa $^{\text {h }}$ Katsuaki Tanaka ${ }^{i}$ Makoto Kako ${ }^{b}$ Wasaburo Koizumia \\ a Department of Gastroenterology, Internal Medicine, Kitasato University School of \\ Medicine, Sagamihara, Japan; 'b Department of Gastroenterology, Shonan Kamakura General \\ Hospital, Kamakura, Japan; ' ${ }^{\prime}$ Gastroenterological Center, Yokohama City University Medical \\ Center, Yokohama, Japan; ' Nakazawa Medical Clinic, Sagamihara, Japan; ${ }^{e}$ Department of \\ Gastroenterology, Shonan Fujisawa Tokushukai Hospital, Fujisawa, Japan; ' Department \\ of Hepatobiliary and Pancreatic Medical Oncology, Kanagawa Cancer Center, Yokohama, \\ Japan; ${ }^{9}$ Division of Gastroenterology and Hepatology, Department of Internal Medicine, \\ St. Marianna University School of Medicine, Kawasaki, Japan; hivision of Gastroenterology \\ and Hepatology, Department of Internal Medicine, Tokai University School of Medicine, \\ Isehara, Japan; 'Department of Gastroenterology, Japanese Red Cross Hadano Hospital, \\ Hadano, Japan
}

\section{Keywords}

Skeletal muscle mass · Hepatocellular carcinoma $\cdot$ Lenvatinib

\begin{abstract}
Background: Low skeletal muscle mass is significantly associated with severe adverse events (AEs) from chemotherapy, and low tolerability leads to decreased survival. We aimed to investigate whether body skeletal muscle mass is correlated with tolerability and prognosis in patients with hepatocellular carcinoma (HCC) treated with lenvatinib. Methods: This multicenter, retrospective study was conducted at five locations in Japan. We included 100 patients with HCC treated with lenvatinib. Skeletal muscle mass was measured by computed tomography and normalized for height in $\mathrm{m}^{2}$ as skeletal muscle index (SMI). The assessment criteria for low SMI were taken from the sarcopenia criteria of the Japan Society of Hepatology. We investigated the influence of low SMI on drug withdrawal due to severe AEs in the first 2 months and on time to treatment failure (TTF) and overall survival (OS). Results: The numbers of high- and low-SMI patients were 41 and 59, respectively. Those with severe AEs leading to withdraw in the high- and low-SMI groups were 7 and 23, respectively. The low-SMI group had a higher
\end{abstract}


withdrawal rate than the high-SMI group ( $p=0.042)$. The median TTF in the low- and highSMI groups was 139 and 230 days, respectively. The median OS in the low- and high-SMI groups was 264 and 353 days, respectively. Patients in the low-SMI group experienced significantly worse OS and TTF than those in the high-SMI group (log-rank test for trend: TTF, $p=0.010 ;$ OS, $p=0.021$ ). Conclusion: Decreased skeletal muscle mass is associated with the occurrence of severe AEs and worse TTF and OS. Skeletal muscle mass can be used as a predictive marker for tolerability and prognosis to lenvatinib in patients with HCC.

(C) 2019 The Author(s)

Published by S. Karger AG, Basel

\section{Introduction}

Hepatocellular carcinoma (HCC) is a common cancer in patients with chronic liver disease and the leading cause of cancer mortality worldwide [1]. Although the principal treatment for HCC is surgical resection, any tumor cells remaining after surgical treatment are a serious health concern for cancer recurrence [2]. Recent advances in the understanding of the molecular pathogenesis of HCC have highlighted the overexpression of growth factors such as vascular endothelial growth factor receptor, fibroblast growth factor, and platelet-derived growth factor receptor [3]. Inhibition of multiple kinases has become an attractive therapeutic approach in patients with HCC without treatment after surgical operation [3-6].

Lenvatinib, an oral multikinase inhibitor that targets vascular endothelial growth factor receptor, platelet-derived growth factor receptor, as well as the oncogenes RAS and RAF, has been shown to improve survival in advanced HCC and is often used for systemic therapy [7, 8]. Based on results from the randomized, open-label, multinational, noninferiority phase III REFLECT trial, lenvatinib was noninferior to sorafenib in terms of overall survival (OS). Furthermore, lenvatinib significantly increased objective response rate, progression-free survival, and time to progression in comparison with sorafenib [9].

Lenvatinib is typically well tolerated by patients, though adverse events (AEs) such as hypertension, diarrhea, decreased appetite, and decreased weight have been reported. However, some patients experience low tolerability to lenvatinib, making it difficult to continue therapy because of debilitating AEs. Serious AEs can lead to decreased survival and higher recurrence/treatment failure rates [9]. Phase I and II studies revealed an inverse relationship between body weight (BW) and plasma lenvatinib levels, with higher lenvatinib levels resulting in decreased BW [7]. Since this trend was more prominent in HCC patients than in patients with other types of solid cancerous tumors, the reduced initial dose based on BW was administered to a number of patients with low BW to improve the safety of lenvatinib in the treatment of patients with HCC [7].

However, BW is not always a reliable metric because it does not distinguish between muscle and fat composition of a person's total mass. Recently, there has been growing evidence that body muscle mass is more accurate in correlation with drug clearance than BW [10-13]. In a study of solid cancer patients, which examined the association between skeletal muscle mass and toxicity from chemotherapy, it was shown that lower skeletal muscle mass was significantly associated with severe AEs [14]. Time to treatment failure (TTF) was also shorter for the lower skeletal muscle mass group compared with the higher skeletal muscle mass group [14].

Based on such findings, skeletal muscle mass may be a good predictor of tolerability and the prognosis to chemotherapy. However, little is known about the clinical impact of skeletal muscle mass in patients with HCC treated with lenvatinib. In the present study, we aimed to investigate whether body skeletal muscle mass was correlated with tolerability and prognosis in patients with advanced HCC treated with lenvatinib. 


\section{Liver Cancer}

Fig. 1. Patient selection criteria and lenvatinib dosages. HCC, hepatocellular carcinoma.

\begin{tabular}{|c|c|}
\hline \multicolumn{2}{|l|}{ Liver Cancer 2020;9:193-206 } \\
\hline DOI: $10.1159 / 000504604$ & $\begin{array}{l}\text { (c) } 2019 \text { The Author(s). Published by S. Karger AG, Basel } \\
\text { www.karger.com/lic }\end{array}$ \\
\hline
\end{tabular}

Uojima et al.: Muscle Mass in Liver Cancer Patients Treated with Lenvatinib

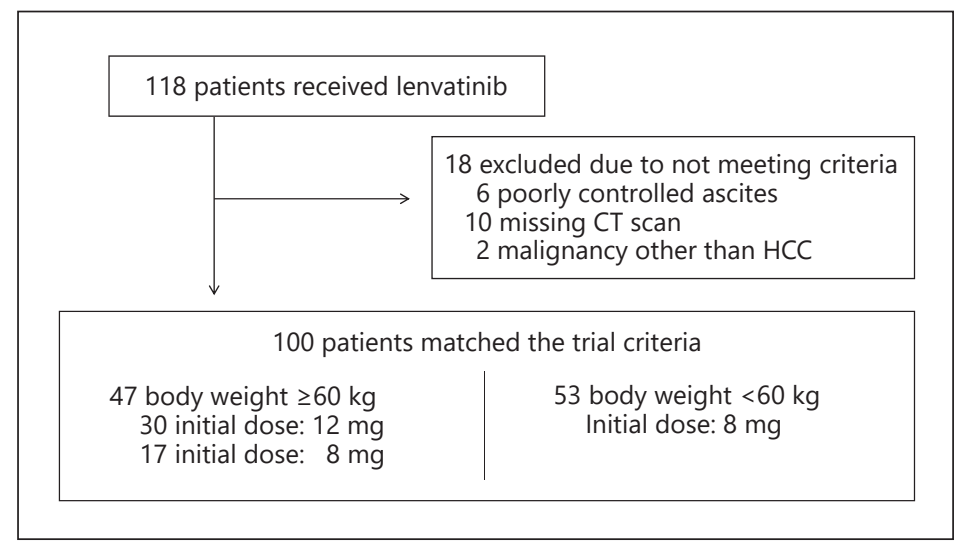

\section{Methods}

\section{Patients}

This multicenter, retrospective study was conducted at five locations in Japan. We included patients with HCC treated with lenvatinib. The diagnosis of liver HCC was based on noninvasive imaging using computed tomography (CT) and/or magnetic resonance imaging. For patients with atypical imaging findings, ultrasound-guided tumor biopsy was conducted for histological assessment. Lenvatinib therapy was recommended for patients with unresectable HCC and (a) presence of distant metastases, (b) refractory response to previous transcatheter arterial therapies for HCC, (c) unsuitability for transcatheter arterial therapies due to anatomical reasons, and (d) vascular invasion such as tumor thrombus in the portal vein. Patients with severe liver function characterized by Child-Pugh class C, poorly controlled ascites, severe renal dysfunction, and malignancy other than HCC were excluded.

A total of 118 patients received lenvatinib from February 2018 to July 5, 2019 (Fig. 1). Of those 118 patients, 18 were excluded because of poorly controlled ascites, a missing CT scan, or a diagnosis of a malignancy other than HCC.

\section{Lenvatinib Dosages}

Patients with no evident risk factors received lenvatinib $12 \mathrm{mg}$ orally once daily for those with a baseline BW $\geq 60 \mathrm{~kg}$ and $8 \mathrm{mg}$ orally once daily for those with a baseline BW $<60 \mathrm{~kg}$ in 28-day cycles. For patients with risk factors based on clinical features, the initial dose administered was reduced from 12 to 8 mg once daily. During administration of treatment, clinicians could adjust the daily dose of lenvatinib according to the frequency and severity of AEs. Lenvatinib was continued until disease progression was implicated, unmanageable AEs occurred, or if patients wished to discontinue treatment at their own discretion. Treatment response was assessed once every 8-12 weeks following the initiation of therapy, and disease progression was evaluated according to the modified Response Evaluation Criteria in Solid Tumors [15].

\section{Measurement of Skeletal Muscle Mass}

The skeletal muscle area was measured by the cross-sectional CT images at the third lumbar vertebra (L3) using the Slice-O-Matic software (version 5.0; Tomovision, Montreal, Canada) on the available CT scan performed before treatment initiation. Skeletal muscle area was normalized for height in $\mathrm{m}^{2}$ and expressed as skeletal muscle index (SMI) (Fig. 2). The skeletal muscles at L3 are the psoas major, the erector spinae, the quadratus lumborum, the rectus abdominis, the transversus abdominis, the internal oblique, and the external oblique. The assessment criteria for low SMI were taken from the working group for creation of sarcopenia assessment criteria of the Japan Society of Hepatology. The cutoff values for low SMI are $<42 \mathrm{~cm}^{2} / \mathrm{m}^{2}$ for men and $<38 \mathrm{~cm}^{2} / \mathrm{m}^{2}$ for women [16].

\section{Clinical Parameters}

Clinical characteristics, including general characteristics, demographic information, Barcelona Clinic Liver Cancer (BCLC) staging for HCC, and all laboratory data, were obtained on the same day for patients who had previously been treated for HCC. Laboratory tests were conducted to measure white blood cells, hemo- 


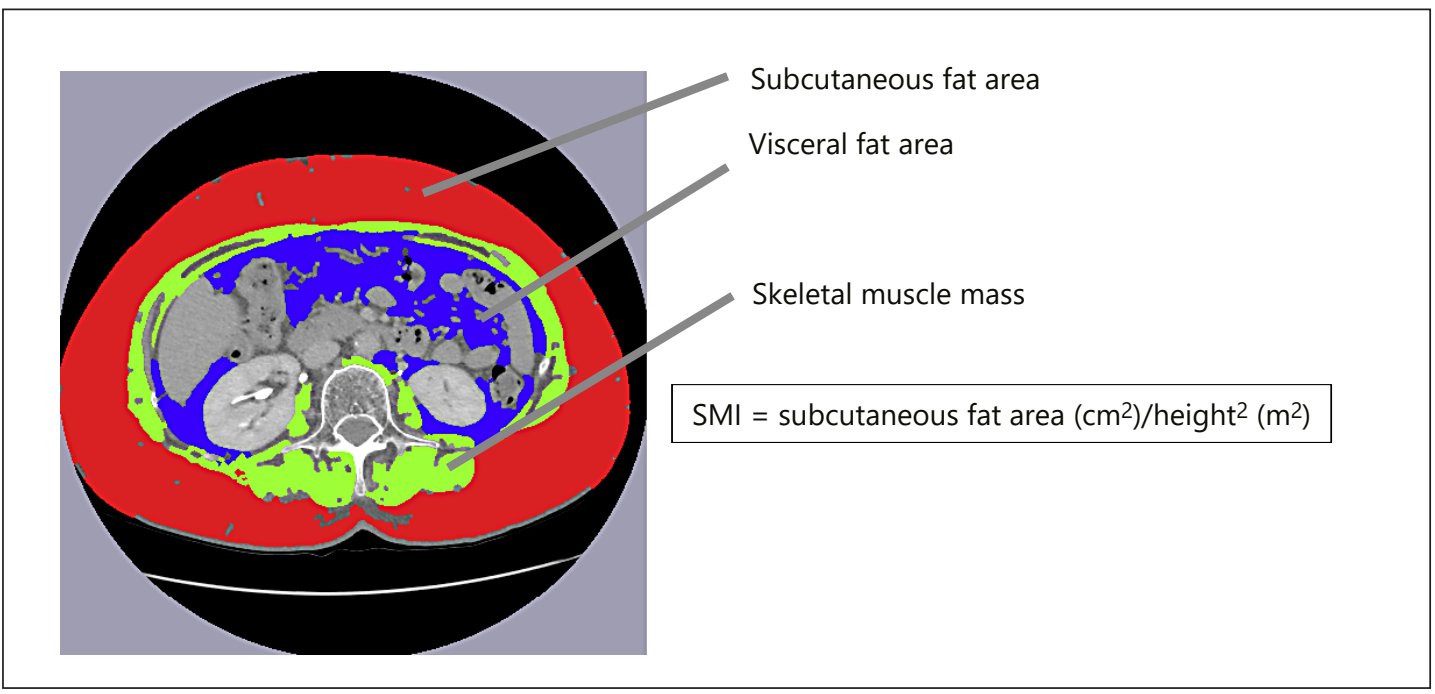

Fig. 2. Cross-sectional areas of skeletal muscle at the third lumbar level were measured by manual tracing on the CT images, and their sum was calculated. The green area indicates skeletal muscle at the third lumbar level. SMI, skeletal muscle index.

globin, platelets, prothrombin time, albumin, blood urea nitrogen, serum creatinine, aspartate aminotransferase, alanine aminotransferase, total bilirubin, serum sodium, serum potassium, total cholesterol, branchedchain amino acid (BCAA) and tyrosine ratio, ammonia, alpha-fetoprotein, and protein induced by vitamin $\mathrm{K}$ absence or antagonist-II.

\section{Primary Endpoint Measurement}

To investigate whether skeletal muscle mass was a useful predictor for tolerability and prognosis in patients treated with lenvatinib, we investigated the influence of low SMI on the occurrence of drug withdrawal due to severe AEs within the first 2 months of therapy. The severity of any serious or nonserious AE was graded using Common Terminology Criteria for Adverse Events, version 4.0. A composite variable of severe AE was defined as any hospitalization and/or grade 3-4 toxicity.

We also investigated the influence of SMI on TTF and OS. TTF was defined as the number of days from chemotherapy initiation to either end of treatment or July 5, 2019. OS was defined as the number of days from chemotherapy initiation to either death or July 5, 2019.

\section{Statistical Analyses}

Data were analyzed using SPSS version 24.0 (IBM Corp., Armonk, NY, USA). All data were expressed as mean \pm standard deviation. Numerical variables were compared using a $t$ test, whereas a paired $t$ test was used for paired data.

Uni- and multivariate analyses with logistic regression models were used to calculate odds ratios and 95\% confidence intervals (CIs) to assess the correlation between severe AEs and clinical characteristics. The Kaplan-Meier method and the log-rank test were used to compare differences in OS and TTF between the two groups. Variables shown by univariate analysis to be significantly associated with TTF and OS were entered into a Cox proportional hazards regression model for multivariate analysis. Differences with a $p$ value $<0.05$ were considered significant. Statistical analyses were performed by Statista Corp., Kyoto, Japan.

\section{Results}

\section{Patients and Baseline Characteristics}

The baseline clinical characteristics of the 100 patients are summarized in Table 1 . The mean age was $71.5 \pm 9.2$ years (range $49-88$ years), and 75 patients $(75.0 \%)$ were male. 


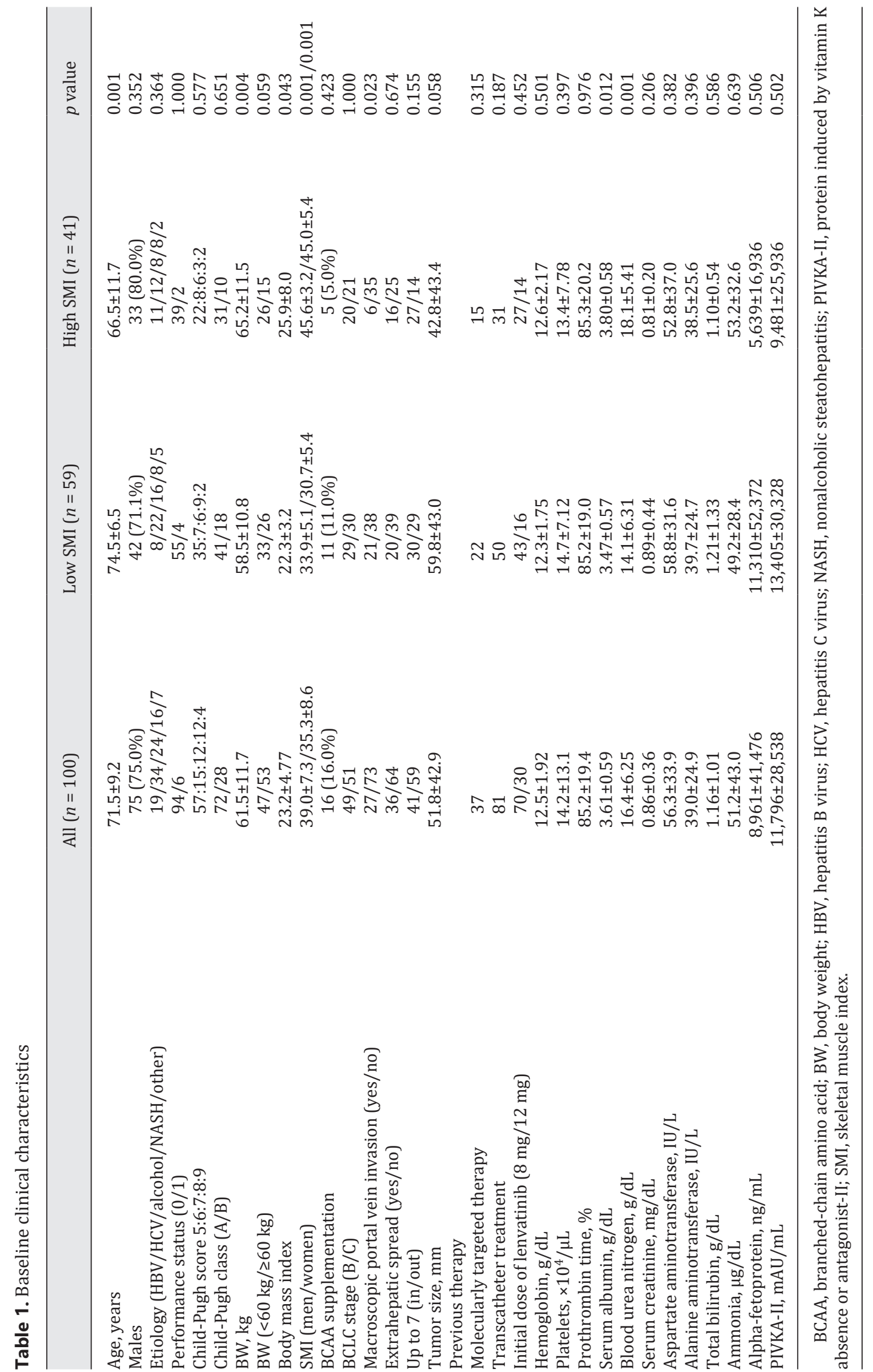




\section{Liver Cancer}

Table 2. Severe adverse events within the first 2 months

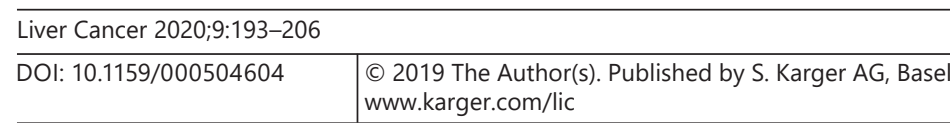

Uojima et al.: Muscle Mass in Liver Cancer Patients Treated with Lenvatinib

\begin{tabular}{lcl}
\hline & $\begin{array}{l}\text { Low SMI } \\
(n=59)\end{array}$ & $\begin{array}{l}\text { High SMI } \\
(n=41)\end{array}$ \\
\hline Decreased appetite & $11(18.6 \%)$ & $2(4.8 \%)$ \\
Decreased weight & $5(8.4 \%)$ & $1(2.4 \%)$ \\
Hepatic encephalopathy & $5(8.4 \%)$ & $1(2.4 \%)$ \\
Hepatic ascites & $4(6.7 \%)$ & $1(2.4 \%)$ \\
Proteinuria & $2(3.4 \%)$ & $1(2.4 \%)$ \\
Diarrhea & $2(3.4 \%)$ & 0 \\
Gastrointestinal bleeding & $1(1.7 \%)$ & $1(2.4 \%)$ \\
Sepsis & $1(1.7 \%)$ & 0 \\
Increased blood bilirubin & $1(1.7 \%)$ & 0 \\
Hypertension & $1(1.7 \%)$ & 0 \\
\hline \multicolumn{1}{c}{ SMI, skeletal muscle index. } & & \\
\hline
\end{tabular}

Seventy-two (72.0\%) and 28 (28.0\%) cases of cirrhosis were noted in Child-Pugh class A and $\mathrm{B}$, respectively. The mean BW was $61.5 \pm 11.7 \mathrm{~kg}$ (range 31.5-97.0 kg), and the mean body mass index was $23.2 \pm 4.77$ (range 14.7-38.3). Chronic liver disease was caused by hepatic $(n=53)$ and nonhepatic viruses $(n=47)$. Forty-nine $(49.0 \%)$ and $51(51.0 \%)$ cases of cirrhosis were noted in BCLC B and C, respectively. Among 36 patients with at least a single site of distant metastatic disease, 27 had macroscopic portal vein invasion.

Regarding the administration of lenvatinib, 17 patients were diagnosed with distant metastases, 37 patients had refractory response to molecularly targeted therapy (sorafenib), 23 patients had refractory response to previous transcatheter arterial therapy, 7 patients were unsuitable for transcatheter arterial therapy due to anatomical reasons, and 16 patients had vascular invasion such as a tumor or thrombus in the portal vein. Regarding the initial dose, 30 patients $(30.0 \%)$ took $12 \mathrm{mg}$, while 70 patients $(70.0 \%)$ took $8 \mathrm{mg}$ once daily. Although there were no patients treated with a reduced initial dose from 4 to $8 \mathrm{mg}$, the initial dose was reduced for 17 patients from 12 to $8 \mathrm{mg}$ once daily because of clinical features - low performance status in 2 patients and Child-Pugh class B in 15 patients.

\section{Clinical Characteristics of Low Skeletal Muscle Mass}

Based on the criteria of the Japan Society of Hepatology, patients were divided into a low-SMI and a high-SMI group. Forty-one patients (41.0\%) were classified as high SMI while 59 patients $(59.0 \%)$ were classified as low SMI. BW and body mass index were lower ( $p=$ 0.004 and $p=0.043$, respectively) in the low-SMI than in the high-SMI group. Age in the low-SMI group was higher than in the high-SMI group. Serum albumin and blood urea nitrogen were lower ( $p=0.012$ and $p=0.001$, respectively) in the low-SMI than in the high-SMI group.

However, the groups did not differ significantly in sex, the cause of liver cirrhosis, ChildPugh score, BCLC stage, hemoglobin level, platelet count, prothrombin time, alanine aminotransferase, aspartate aminotransferase, total bilirubin, creatinine, alpha-fetoprotein, or protein induced by vitamin $\mathrm{K}$ absence or antagonist-II. The mean SMI of all patients, men and women, was $38.1 \pm 7.75,39.0 \pm 7.27$, and $35.3 \pm 8.61$, respectively. The correlation between SMI and BW was moderately positive $(r=0.423, p=0.001)$. In the low-SMI group there were 33 and 26 patients $<60 \mathrm{~kg}$ and $\geq 60 \mathrm{~kg}$, respectively.

\section{Relationship between SMI and Toxicity}

The numbers and proportions of complete response, partial response, stable disease, disease progression, and unevaluated response in 2 months were 3 (3.0\%), 28 (28.0\%), 32 
Table 3. Univariate analysis of factors affecting severe adverse events

\begin{tabular}{|c|c|c|c|}
\hline Variable & & OR $(95 \%$ CI) & $p$ value \\
\hline SMI & $\begin{array}{l}\text { 1: high } \\
\text { 2: low }\end{array}$ & $3.333(1.269-8.744)$ & 0.015 \\
\hline Age, years & $\begin{array}{l}1:<75 \\
2: \geq 75\end{array}$ & 1.759 (0.737-4.199) & 0.203 \\
\hline Male sex & $\begin{array}{l}1:- \\
2:+\end{array}$ & $2.122(0.714-6.308)$ & 0.176 \\
\hline BW, kg & $\begin{array}{l}1:<60 \\
2: \geq 60\end{array}$ & $1.800(0.764-4.243)$ & 0.179 \\
\hline Body mass index & $\begin{array}{l}1:<23 \\
2: \geq 23\end{array}$ & $1.113(0.475-2.606)$ & 0.805 \\
\hline Serum creatinine, mg/dL & $\begin{array}{l}1:<1.0 \\
2: \geq 1.0\end{array}$ & 4.211 (1.539-11.51) & 0.005 \\
\hline ALBI grade & $\begin{array}{l}1: \leq-2.60 \\
2: \leq-1.39 \\
3:>-1.39 \\
\end{array}$ & $\begin{array}{l}1.738(0.679-4.452) \\
3.259(0.683-15.54)\end{array}$ & $\begin{array}{l}0.249 \\
0.138\end{array}$ \\
\hline Child-Pugh class & $\begin{array}{l}\text { 1: A } \\
2: \mathrm{B}\end{array}$ & 3.235 (1.291-8.110) & 0.012 \\
\hline
\end{tabular}

ALBI, albumin-bilirubin; BW, body weight; CI, confidence interval; OR, odds ratio; SMI, skeletal muscle index.

(32.0\%), 17 (17.0\%), and 20 (20.0\%), respectively. All unevaluated patients died before they could be assessed with CT and/or magnetic resonance imaging.

Treatment-related AEs within the first 2 months of the study period led to a lenvatinib dose reduction in 51 patients and complete drug withdrawal in 30 patients (Table 2). Of those 30 patients who experienced severe AEs leading to complete withdrawal, $17.1 \%(n=7)$ were in the low-SMI group and $45.1 \%(n=23)$ in the high-SMI group. The low-SMI group had a significantly higher withdrawal rate than the high-SMI group $(p=0.042)$. By contrast, there were no significant differences in BW between the patients who continued lenvatinib treatment and those who decided to stop taking the drug.

In the low-SMI group, severe AEs were decreased appetite $(n=11)$, decreased BW $(n=$ $5)$, hepatic encephalopathy $(n=5)$, ascites $(n=4)$, proteinuria $(n=2)$, diarrhea $(n=2)$, increased blood bilirubin $(n=1)$, hypertension $(n=1)$, sepsis $(n=1)$, and gastrointestinal bleeding $(n=1)$. In the high-SMI group, severe AEs were decreased appetite $(n=2)$, proteinuria $(n=1)$, decreased weight $(n=1)$, hepatic encephalopathy $(n=1)$, ascites $(n=1)$, and gastrointestinal bleeding $(n=1)$ (Table 2).

We also evaluated the relationship between severe AEs during the first 2 months and the baseline clinical characteristics in a logistic regression model (Table 3). Univariate analysis confirmed that SMI, serum creatinine, and Child-Pugh class were independent from the factors for the occurrence of severe AEs. Multivariate analysis was not applicable because of small sample size.

\section{Influence of Low Skeletal Muscle Mass on TTF}

The median TTF in all enrolled patients was 179 days (95\% CI 147-212 days). Lenvatinib treatment was discontinued for one of three reasons: disease progression, severe unman- 


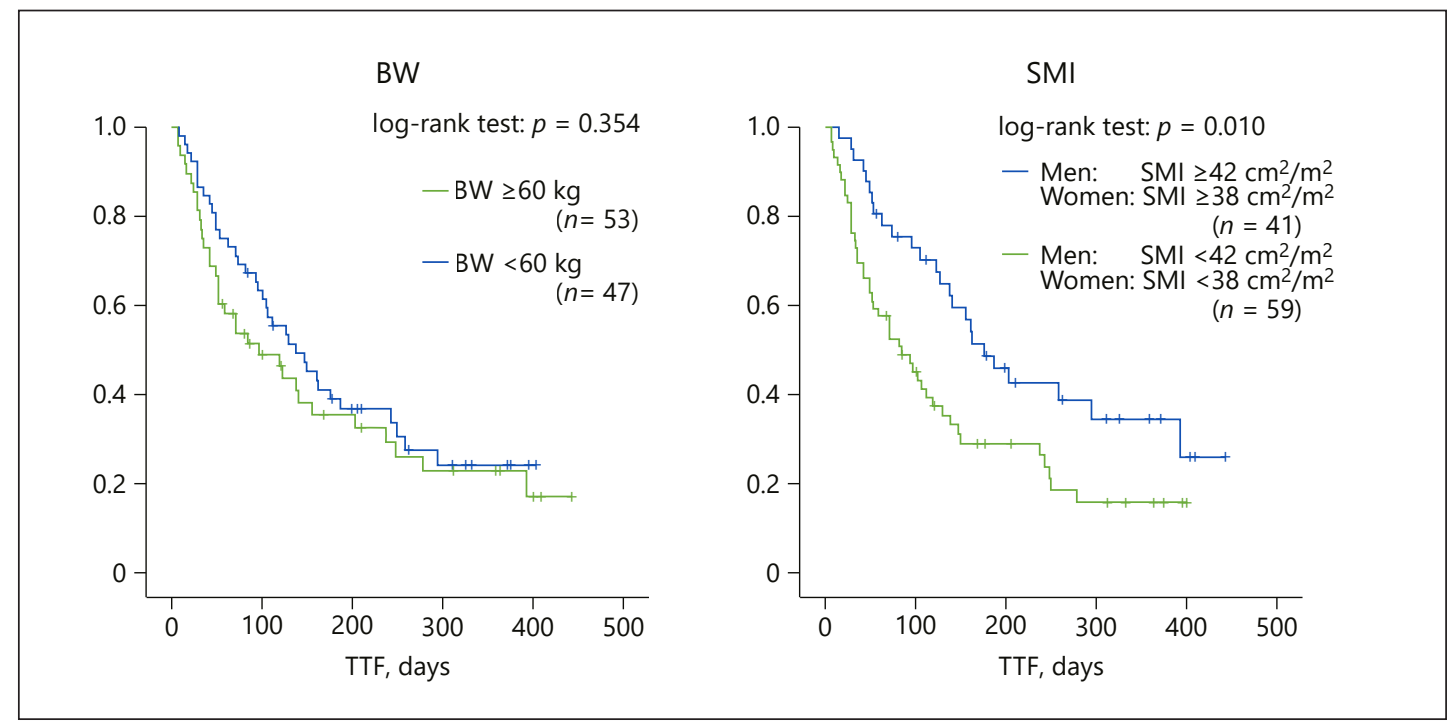

Fig. 3. TTF of patients in the low- and high-SMI groups. Patients with a low SMI had a shorter median TTF (139 days) compared to patients with a high SMI (230 days) (log rank $p=0.010)$. BW, body weight; SMI, skeletal muscle index; TTF, time to treatment failure.

ageable AEs, or patient decision to discontinue treatment. Twenty-one patients stopped treatment due to disease progression, 10 decided to discontinue treatment on their own discretion, and 48 stopped due to severe AEs.

The median TTF in the low- and high-SMI groups was 139 and 230 days (95\% CI 102-175 days and 179-281 days), respectively. Patients in the low-SMI group experienced significantly worse TTF than those in the high-SMI group (log-rank test for trend: TTF, $p=0.010$ ) (Fig. 3). By contrast, there were no significant differences in TTF between the patients whose BW was $<60 \mathrm{~kg}$ and those with a BW of $\geq 60 \mathrm{~kg}$ (log-rank test for trend: TTF, $p=0.354$ ). These results suggest that SMI is a better indicator of TTF than BW.

In uni- and multivariate analysis, factors significantly associated with poor TTF included SMI and albumin-bilirubin grade (Table 4). Specifically, the hazard ratio of low SMI for TTF was 1.716 (95\% CI 1.041-2.827, $p=0.034)$.

\section{Clinical Course after Administration of Lenvatinib}

Treatments after discontinuance of lenvatinib therapy were best supportive care $(n=$ $44)$, administration of another molecularly targeted therapy $(n=10)$, radiation therapy $(n=$ 4 ), and transcatheter treatment $(n=12)$. For 37 patients with a history of molecularly targeted therapy, we selected best supportive care $(n=31)$, transcatheter treatment $(n=3)$, radiation therapy $(n=2)$, and administration of another molecularly targeted therapy $(n=1)$.

\section{Influence of Low Skeletal Muscle Mass on OS}

The median OS in all patients, the low-SMI group, and the high-SMI group was 301, 264, and 353 days (95\% CI 267-335, 220-308, and 304-401 days), respectively. Patients in the low-SMI group experienced significantly worse OS than those in the high-SMI group (log-rank test for trend: OS, $p=0.021$ ) (Fig. 4). In contrast, there was no significant difference in OS between the patients in the low-BW group and the high-BW group.

In uni- and multivariate analysis, the factors significantly associated with poor OS included SMI, albumin-bilirubin grade, and alpha-fetoprotein (Table 5). Specifically, the hazard ratio of low SMI for OS was 2.246 (95\% CI 1.091-4.623, $p=0.028$ ). 


\section{Liver Cancer}

\begin{tabular}{|c|c|}
\hline \multicolumn{2}{|l|}{ Liver Cancer 2020;9:193-206 } \\
\hline DOI: $10.1159 / 000504604$ & $\begin{array}{l}\text { (c) } 2019 \text { The Author(s). Published by S. Karger AG, Basel } \\
\text { www.karger.com/lic }\end{array}$ \\
\hline
\end{tabular}

Uojima et al.: Muscle Mass in Liver Cancer Patients Treated with Lenvatinib

Table 4. Uni- and multivariate analyses of factors affecting time to treatment failure

\begin{tabular}{|c|c|c|c|c|c|}
\hline \multirow[t]{2}{*}{ Variable } & & \multicolumn{2}{|l|}{ Univariate analysis } & \multicolumn{2}{|l|}{ Multivariate analysis } \\
\hline & & OR $(95 \% \mathrm{CI})$ & $p$ value & OR $(95 \% \mathrm{CI})$ & $p$ value \\
\hline SMI & $\begin{array}{l}\text { 1: high } \\
2 \text { : low }\end{array}$ & 1.888 (1.154-3.089) & 0.011 & $1.716(1.041-2.827)$ & 0.034 \\
\hline Age, years & $\begin{array}{l}1:<75 \\
2: \geq 75\end{array}$ & $1.306(0.747-2.284)$ & 0.348 & & \\
\hline Male sex & $\begin{array}{l}1:- \\
2:+\end{array}$ & $1.533(0.879-1.790)$ & 0.128 & & \\
\hline BW, kg & $\begin{array}{l}1:<60 \\
2: \geq 60\end{array}$ & 1.247 (0.779-1.997) & 0.357 & & \\
\hline BCLC stage & $\begin{array}{l}\text { 1: B } \\
2: \mathrm{C}\end{array}$ & $1.228(0.766-1.970)$ & 0.394 & & \\
\hline BCAA supplementation & $\begin{array}{l}1:- \\
2:+\end{array}$ & $1.635(0.891-2.998)$ & 0.112 & & \\
\hline $\begin{array}{l}\text { Previous therapy: } \\
\text { transcatheter treatment }\end{array}$ & $\begin{array}{l}1:- \\
2:+\end{array}$ & $1.094(0.609-1.967)$ & 0.763 & & \\
\hline $\begin{array}{l}\text { Previous therapy: } \\
\text { molecularly targeted therapy }\end{array}$ & $\begin{array}{l}1:- \\
2:+\end{array}$ & $1.376(0.857-2.207)$ & 0.186 & & \\
\hline $\begin{array}{l}\text { Refractory to } \\
\text { transcatheter treatment }\end{array}$ & $\begin{array}{l}1:- \\
2:+\end{array}$ & $1.003(0.626-1.607)$ & 0.990 & & \\
\hline Alpha-fetoprotein, ng/mL & $\begin{array}{l}1:<200 \\
2: \geq 200\end{array}$ & $1.365(0.849-2.197)$ & 0.199 & & \\
\hline ALBI grade & $\begin{array}{l}1: \leq-2.60 \\
2: \leq-1.39 \\
3:>-1.39\end{array}$ & $\begin{array}{l}2.000(1.163-3.439) \\
3.942(1.756-8.850)\end{array}$ & $\begin{array}{l}0.012 \\
0.001\end{array}$ & $1.817(1.047-3.151)$ & 0.034 \\
\hline
\end{tabular}

ALBI, albumin-bilirubin; BCAA, branched-chain amino acid; BCLC, Barcelona Clinic Liver Cancer; BW, body weight; CI, confidence interval; OR, odds ratio; SMI, skeletal muscle index.

\section{Influence of Low Skeletal Muscle Mass in Child-Pugh Class A}

We also analyzed 72 patients with Child-Pugh class A who exhibited good indications for treatment with lenvatinib. Forty-one patients were classified as low SMI and 31 as high SMI. The median TTF in the low- and high-SMI groups was 158 and 267 days (95\% CI 115-201 and 208-327 days), respectively. The median OS in the low- and high-SMI groups was 304 and 390 days (95\% CI 253-354 and 343-438 days), respectively. Patients in the low-SMI group experienced significantly worse TTF than those in the high-SMI group (log-rank test for trend: TTF, $p=0.011$; OS, $p=0.041$ ).

\section{Discussion}

This study showed that skeletal muscle mass was a good predictor for drug toxicity in patients with HCC who were treated with lenvatinib. This result is important with respect to making treatment decisions for HCC patients. Previous phase I and II studies revealed that the reduced initial dose based on BW improved the safety of lenvatinib in the treatment of patients with HCC [7]. As a result, there were no significant differences in drug withdrawal 


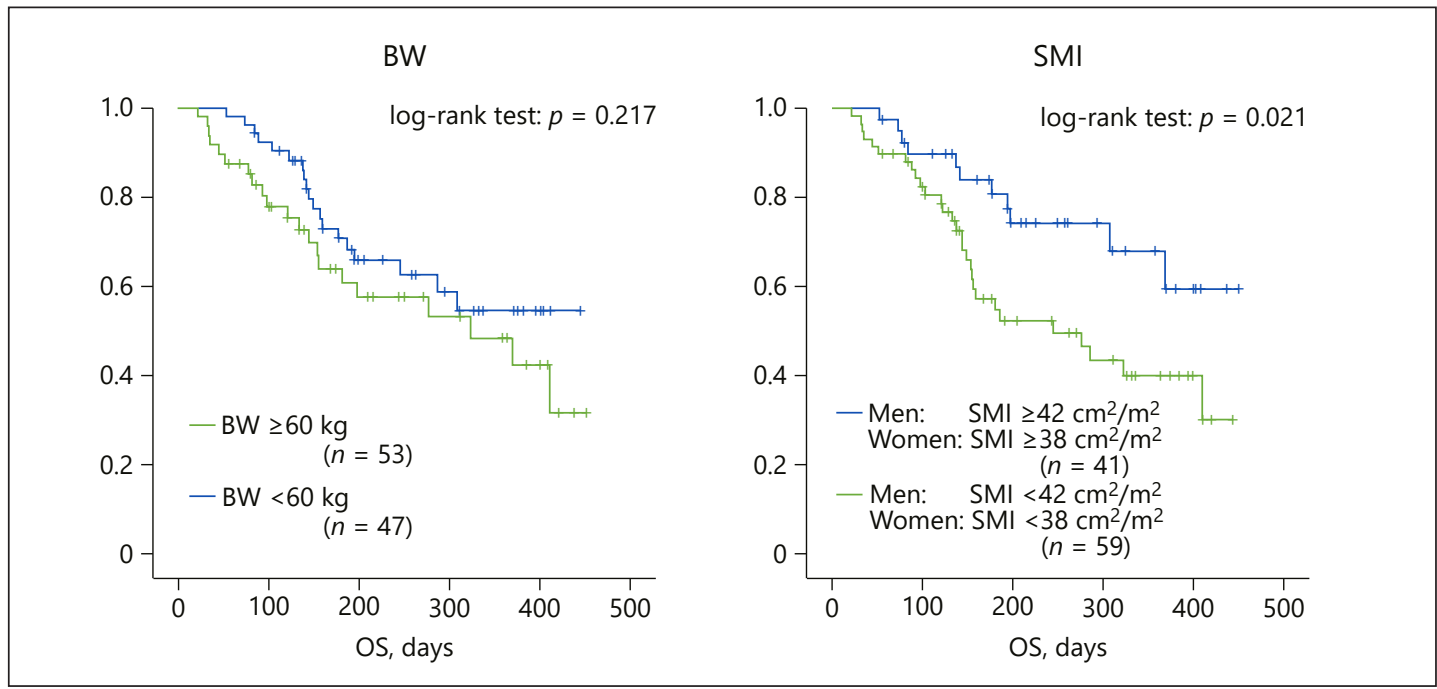

Fig. 4. OS of patients in the low- and high-SMI groups. Patients with a low SMI had a shorter median OS (264 days) compared to patients with a high SMI (353 days) (log rank $p=0.021$ ). BW, body weight; OS, overall survival; SMI, skeletal muscle index.

in 2 months between patients who had a low BW $(<60 \mathrm{~kg})$ and those who had a high BW $(\geq 60 \mathrm{~kg}$ ). However, the study indicated that many patients (30\%) stopped chemotherapy due to treatment-related severe AEs notwithstanding a relatively short treatment span. Since the reduced initial dose based on BW may be insufficient for patients with HCC treated with lenvatinib in our data, a predictive marker for toxicity is warranted. Skeletal muscle mass measurements using CT images may help in the assessment of treatment risks and in the determination of individualized chemotherapy dosing [17].

Our study also revealed that patients with low SMI experienced significantly worse OS and TTF than those with high SMI. Although age and macroscopic portal vein invasion in the high-SMI group were higher than in the low-SMI group, multivariate analysis showed that low SMI was an independent risk factor for short OS and TTF. It is well known that low tolerability of chemotherapy leads to decreased survival and higher recurrence/treatment failure rates $[18,19]$. Previous studies have reported that low skeletal muscle attenuation was associated with higher toxicity and shorter TTF in patients receiving chemotherapy for solid tumors [10-13]. In support of these previous studies, our results indicate that skeletal muscle mass measurement may prove to be the most important indicator for chemotherapy toxicity and could have important implications for treatment decisions.

Skeletal muscle wasting was frequently observed in patients with advanced cancer. In a recent meta-analysis, 19-74\% of patients with advanced solid tumors were sarcopenic [14]. This trend was more prominent in HCC patients than in patients with other types of solid cancer because patients with advanced HCC have many risk factors for skeletal muscle wasting, such as protein energy malnutrition and being elderly. The liver is an essential organ involved in protein, fat, and carbohydrate metabolism as well as energy generation [20]. Progression of liver fibrosis and/or a functional liver disorder leads to a reduction in glycogen storage and an increase in the consumption of protein as the primary material. Therefore, the incidence of protein energy malnutrition is high in patients with liver cirrhosis.

An epidemiological characteristic of HCC in Japan was also the risk factor of skeletal muscle wasting in patients with advanced HCC. As lifespans increase in Japan, HCC diagnoses in the elderly are becoming more prevalent. Aging is a risk factor for skeletal muscle wasting. 
Uojima et al.: Muscle Mass in Liver Cancer Patients Treated with Lenvatinib

Table 5. Uni- and multivariate analyses of factors affecting overall survival

\begin{tabular}{|c|c|c|c|c|c|}
\hline \multirow[t]{2}{*}{ Variable } & & \multicolumn{2}{|l|}{ Univariate analysis } & \multicolumn{2}{|l|}{ Multivariate analysis } \\
\hline & & OR $(95 \% \mathrm{CI})$ & $p$ value & OR $(95 \% \mathrm{CI})$ & $p$ value \\
\hline SMI & $\begin{array}{l}\text { 1: high } \\
\text { 2: low }\end{array}$ & $2.218(1.106-4.449)$ & 0.025 & $2.246(1.091-4.623)$ & 0.028 \\
\hline Age, years & $\begin{array}{l}1:<75 \\
2: \geq 75\end{array}$ & $1.046(0.537-2.035)$ & 0.896 & & \\
\hline Male sex & $\begin{array}{l}1:- \\
2:+\end{array}$ & $1.400(0.722-2.715)$ & & & \\
\hline BW, kg & $\begin{array}{l}1:<60 \\
2: \geq 60\end{array}$ & $1.477(0.792-2.755)$ & 0.220 & & \\
\hline BCLC stage & $\begin{array}{l}\text { 1: B } \\
2: \mathrm{C}\end{array}$ & $1.741(0.916-3.306)$ & 0.090 & & \\
\hline BCAA supplementation & $\begin{array}{l}1:- \\
2:+\end{array}$ & $1.235(0.544-2.800)$ & 0.614 & & \\
\hline $\begin{array}{l}\text { Previous therapy: } \\
\text { transcatheter treatment }\end{array}$ & $\begin{array}{l}1:- \\
2:+\end{array}$ & $1.308(0.548-3.124)$ & 0.546 & & \\
\hline $\begin{array}{l}\text { Previous therapy: } \\
\text { molecularly targeted therapy }\end{array}$ & $\begin{array}{l}1:- \\
2:+\end{array}$ & $1.543(0.784-3.037)$ & 0.209 & & \\
\hline $\begin{array}{l}\text { Refractory to } \\
\text { transcatheter treatment }\end{array}$ & $\begin{array}{l}1:- \\
2:+\end{array}$ & $1.224(0.657-2.280)$ & 0.524 & & \\
\hline Alpha-fetoprotein, ng/mL & $\begin{array}{l}1:<200 \\
2: \geq 200\end{array}$ & $2.193(1.161-4.142)$ & 0.016 & $2.335(1.210-4.507)$ & 0.011 \\
\hline ALBI score & $\begin{array}{l}1: \leq-2.60 \\
2: \leq-1.39 \\
3:>-1.39\end{array}$ & $\begin{array}{l}2.820(1.293-6.154) \\
3.418(1.425-8.198)\end{array}$ & $\begin{array}{l}0.009 \\
0.006\end{array}$ & $4.636(1.086-5.837)$ & 0.031 \\
\hline
\end{tabular}

ALBI, albumin-bilirubin; BCAA, branched-chain amino acid; BCLC, Barcelona Clinic Liver Cancer; BW, body weight; CI, confidence interval; OR, odds ratio; SMI, skeletal muscle index.

Skeletal muscle mass decreases by approximately $1 \%$ each year after the age of 50 [21], and $80 \%$ of the patients in the present study were older than 50 years.

Furthermore, treatment history, such as molecularly targeted therapy and transcatheter treatment, may lead to sarcopenia. Especially, this trend will probably be obvious for patients whose diseases progress on molecularly targeted therapy and transcatheter arterial chemoembolization [22]. Although researchers have already reported that skeletal muscle loss was related to AEs, TTF, and OS in patients with HCC treated with sorafenib [18], skeletal muscle mass for lenvatinib therapy after refractory responses to sorafenib is clinically more significant.

It is reported that early recognition and treatment of skeletal muscle loss has the potential to bring additional benefits for patients scheduled to receive chemotherapy [22]. Therefore, a strategy to reduce toxicity and increase or prevent further loss of muscle mass is required in HCC patients who received lenvatinib. There are currently effective reports for non pharmacological treatments such as nutritional therapy and exercise training [23, 24]. Especially, BCAA and carnitine supplementation may be a useful strategy to improve muscle mass and reduce drug toxicity for patients with chronic liver disease and cancer cachexia [25]. Koya et al. [26] reported that exercise with BCAA supplementation prevented skeletal muscle atrophy during 
Uojima et al.: Muscle Mass in Liver Cancer Patients Treated with Lenvatinib

treatment of HCC in patients with chronic liver disease. Ohara et al. [27] also reported that carnitine supplementation significantly led to suppression of skeletal muscle loss in patients with HCC. Although BCAA supplementation was not related to OS or TTF in the present study, we administered BCAA supplementation in later stages of therapy to sarcopenic patients. Therefore, for lenvatinib therapy to be effective, physicians should seek to intervene at an early stage to prevent skeletal muscle loss from falling below the minimum muscle mass levels.

The present study has some limitations. First, the sample size was relatively small. Furthermore, the sample selection was biased because 18 patients were excluded according to the exclusion criteria. Therefore, further studies are warranted to re-examine these results in another cohort to draw definitive conclusions. Second, tumor biopsy was not carried out in every case to assess the degree of HCC. Third, we investigated the influence of low SMI on the occurrence of grade 3-4 toxicity; however, it was difficult to evaluate grade 1-2 toxicity because of this being a multisite study. Finally, we evaluated the quantity of skeletal muscle but could not evaluate the quality of muscle, which are both indicators of the prognosis in patients with HCC. Future studies on the quality of skeletal muscle mass could make it become a prognostic marker for the tolerability of other cancer therapeutics.

In conclusion, we found that high skeletal muscle mass is correlated with increased lenvatinib tolerability in patients with advanced HCC. We also found that low muscle mass is a risk factor for short TTF and OS in patients with HCC. Collectively, our findings support the need to evaluate skeletal muscle mass as an indicator for chemotherapy tolerability and survival prediction in patients with HCC treated with lenvatinib.

\section{Acknowledgment}

We thank Robert E. Brandt, Founder, CEO, and CME of MedEd Japan, for editing and formatting the manuscript.

\section{Statement of Ethics}

This study was approved by the institutional review board ethics committees at all institutes, and the protocol for this study conforms to the provisions of the Declaration of Helsinki. This study is registered in the UMIN Clinical Trials Registry as UMIN000037380. It is a retrospective observational study. Informed consent was obtained from all individual participants included in the study by the opt-out method.

\section{Disclosure Statement}

The authors have no conflicts of interest.

\section{Funding Sources}

None.

\section{Author Contributions}

Conception and design, collection and assembly of data, and statistical analysis: H. Uojima. Collection and assembly of data: M. Chuma, Y. Tanaka, and K. Ogushi. Data analysis and interpretation: H. Hidaka and T. Nakazawa. General supervision of the study group: M. Kako and K. Tanaka. Writing and final approval of the manuscript: all authors. 
Uojima et al.: Muscle Mass in Liver Cancer Patients Treated with Lenvatinib

\section{Data Availability}

The technical appendix, statistical code, and dataset are available from the corresponding author.

\section{References}

1 Forner A, Reig M, Bruix J. Hepatocellular carcinoma. Lancet. 2018 Mar;391(10127):1301-14.

2 Personeni N, Rimassa L. Hepatocellular carcinoma: a global disease in need of individualized treatment strategies. J Oncol Pract. 2017 Jun;13(6):368-9.

3 Cheng AL, Kang YK, Chen Z, Tsao CJ, Qin S, Kim JS, et al. Efficacy and safety of sorafenib in patients in the AsiaPacific region with advanced hepatocellular carcinoma: a phase III randomised, double-blind, placebocontrolled trial. Lancet Oncol. 2009 Jan;10(1):25-34.

4 Johnson PJ, Qin S, Park JW, Poon RT, Raoul JL, Philip PA, et al. Brivanib versus sorafenib as first-line therapy in patients with unresectable, advanced hepatocellular carcinoma: results from the randomized phase III BRISK-FL study. J Clin Oncol. 2013 Oct;31(28):3517-24.

5 Abe M, Miyake T, Kuno A, Imai Y, Sawai Y, Hino K, et al. Association between Wisteria floribunda agglutininpositive Mac-2 binding protein and the fibrosis stage of non-alcoholic fatty liver disease. J Gastroenterol. 2015 Jul;50(7):776-84.

6 Bruix J, Qin S, Merle P, Granito A, Huang YH, Bodoky G, et al.; RESORCE Investigators. Regorafenib for patients with hepatocellular carcinoma who progressed on sorafenib treatment (RESORCE): a randomised, doubleblind, placebo-controlled, phase 3 trial. Lancet. 2017 Jan;389(10064):56-66.

7 Ikeda K, Kudo M, Kawazoe S, Osaki Y, Ikeda M, Okusaka T, et al. Phase 2 study of lenvatinib in patients with advanced hepatocellular carcinoma. J Gastroenterol. 2017 Apr;52(4):512-9.

8 Ikeda M, Okusaka T, Mitsunaga S, Ueno H, Tamai T, Suzuki T, et al. Safety and pharmacokinetics of lenvatinib in patients with advanced hepatocellular carcinoma. Clin Cancer Res. 2016 Mar;22(6):1385-94.

9 Kudo M, Finn RS, Qin S, Han KH, Ikeda K, Piscaglia F, et al. Lenvatinib versus sorafenib in first-line treatment of patients with unresectable hepatocellular carcinoma: a randomised phase 3 non-inferiority trial. Lancet. 2018 Mar;391(10126):1163-73.

10 McLeay SC, Morrish GA, Kirkpatrick CM, Green B. The relationship between drug clearance and body size: systematic review and meta-analysis of the literature published from 2000 to 2007. Clin Pharmacokinet. 2012 May; 51(5):319-30.

11 Shachar SS, Deal AM, Weinberg M, Nyrop KA, Williams GR, Nishijima TF, et al. Skeletal muscle measures as predictors of toxicity, hospitalization, and survival in patients with metastatic breast cancer receiving taxanebased chemotherapy. Clin Cancer Res. 2017 Feb;23(3):658-65.

12 Prado CM, Baracos VE, McCargar LJ, Reiman T, Mourtzakis M, Tonkin K, et al. Sarcopenia as a determinant of chemotherapy toxicity and time to tumor progression in metastatic breast cancer patients receiving capecitabine treatment. Clin Cancer Res. 2009 Apr;15(8):2920-6.

13 Kudo M. Lenvatinib may drastically change the treatment landscape of hepatocellular carcinoma. Liver Cancer. 2018 Mar; 7(1):1-19.

14 Shachar SS, Williams GR, Muss HB, Nishijima TF. Prognostic value of sarcopenia in adults with solid tumours: A meta-analysis and systematic review. Eur J Cancer. 2016 Apr;57:58-67.

15 Lencioni R, Llovet JM. Modified RECIST (mRECIST) assessment for hepatocellular carcinoma. Semin Liver Dis. 2010 Feb;30(1):52-60.

16 Nishikawa H, Shiraki M, Hiramatsu A, Moriya K, Hino K, Nishiguchi S. Japan Society of Hepatology guidelines for sarcopenia in liver disease (1st edition): recommendation from the working group for creation of sarcopenia assessment criteria. Hepatol Res. 2016 Sep;46(10):951-63.

17 Harimoto N, Shirabe K, Yamashita YI, Ikegami T, Yoshizumi T, Soejima Y, et al. Sarcopenia as a predictor of prognosis in patients following hepatectomy for hepatocellular carcinoma. Br J Surg. 2013 Oct; 100(11):152330.

18 Hiraoka A, Hirooka M, Koizumi Y, Izumoto H, Ueki H, Kaneto M, et al. Muscle volume loss as a prognostic marker in hepatocellular carcinoma patients treated with sorafenib. Hepatol Res. 2017 May;47(6):558-65.

19 Tamandl D, Paireder M, Asari R, Baltzer PA, Schoppmann SF, Ba-Ssalamah A. Markers of sarcopenia quantified by computed tomography predict adverse long-term outcome in patients with resected oesophageal or gastro-oesophageal junction cancer. Eur Radiol. 2016 May;26(5):1359-67.

20 Hiraoka A, Michitaka K, Ueki H, Kaneto M, Aibiki T, Okudaira T, et al. Sarcopenia and two types of presarcopenia in Japanese patients with chronic liver disease. Eur J Gastroenterol Hepatol. 2016 Aug;28(8):940-7.

21 Keller K, Engelhardt M. Strength and muscle mass loss with aging process. Age and strength loss. Muscles Ligaments Tendons J. 2014 Feb;3(4):346-50.

22 Fujita M, Takahashi A, Hayashi M, Okai K, Abe K, Ohira H. Skeletal muscle volume loss during transarterial chemoembolization predicts poor prognosis in patients with hepatocellular carcinoma. Hepatol Res. 2019 Jul; 49(7):778-86.

23 Mitchell WK, Williams J, Atherton P, Larvin M, Lund J, Narici M. Sarcopenia, dynapenia, and the impact of advancing age on human skeletal muscle size and strength; a quantitative review. Front Physiol. 2012 Jul;3:260. 
24 Corcoran MP, Nelson ME, Sacheck JM, Reid KF, Kirn D, Fielding RA, et al. Efficacy of an exercise and nutritional supplement program on physical performance and nutritional status in older adults with mobility limitations residing at senior living facilities. J Aging Phys Act. 2017 Jul;25(3):453-63.

25 Uojima H, Sakurai S, Hidaka H, Kinbara T, Sung JH, Ichita C, et al. Effect of branched-chain amino acid supplements on muscle strength and muscle mass in patients with liver cirrhosis. Eur J Gastroenterol Hepatol. 2017 Dec;29(12):1402-7.

26 Koya S, Kawaguchi T, Hashida R, Goto E, Matsuse H, Saito H, et al. Effects of in-hospital exercise on liver function, physical ability, and muscle mass during treatment of hepatoma in patients with chronic liver disease. Hepatol Res. 2017 Mar;47(3):E22-34.

27 Ohara M, Ogawa K, Suda G, Kimura M, Maehara O, Shimazaki T, et al. L-carnitine suppresses loss of skeletal muscle mass in patients with liver cirrhosis. Hepatol Commun. 2018 Aug;2(8):906-18. 